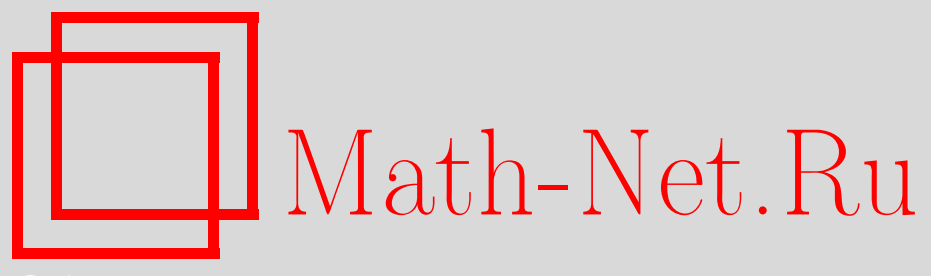

В. С. Атабекян, Равномерная неаменабельность подгрупп свободных бернсайдовых групп нечетного периода, Матем. заметки, 2009, том 85, выпуск 4, 516-523

DOI: https://doi.org/10.4213/mzm4890

Использование Общероссийского математического портала Math-Net.Ru подразумевает, что вы прочитали и согласны с пользовательским соглашением http://www . mathnet.ru/rus/agreement

Параметры загрузки:

IP : 54.198 .187 .58

26 апреля 2023 г., 15:46:54

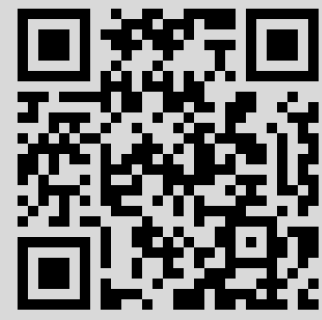


Том 85 выпуск 4 апрель 2009

УДК 512.543

\section{Равномерная неаменабельность подгрупп свободных бернсайдовых групп нечетного периода}

\section{В. С. Атабекян}

Известная теорема С. И. Адяна утверждает, что для любого $m \geqslant 2$ и нечетного $n \geqslant 665$ свободная $m$-порожденная бернсайдовая группа $B(m, n)$ периода $n$ неаменабельная. В работе доказывается, что каждая нециклическая подгруппа свободной бернсайдовой группы $B(m, n)$ нечетного периода $n \geqslant 1003$ является равномерно неаменабельной группой. Из этого результата для нечетных $n \geqslant 1003$ следует положительный ответ на вопрос де ля Арпа: имеют ли бесконечные свободные бернсайдовые группы $B(m, n)$ равномерно экспоненциальный рост? Доказывается также, что в каждом $S$-шаре радиуса $(400 n)^{3}$ содержатся два элемента, которые являются базисом свободной периодической подгруппы ранга 2 группы $B(m, n)$, где $S$ - произвольное множество элементов, порождающих нециклическую подгруппу группы $B(m, n)$.

Библиография: 21 название.

Пусть $A$ - конечное подмножество группы $G$, а $S$ - конечное порождающее множество группы $G$. Границей подмножества $A$ относительно конечного порождающего множества $S \subset G$ называется множество

$$
\partial_{S}(A) \rightleftharpoons\left\{a \in A \mid a x \notin A \text { для некоторого } x \in S^{ \pm 1}\right\} .
$$

ОПРЕДЕЛЕНИЕ 1. Константой Фелнера группъ $G$ относителъно порождающего множества $S$ называется число

$$
\mathrm{F} \mathrm{l}_{S}(G) \rightleftharpoons \inf _{A} \frac{\left|\partial_{S}(A)\right|}{|A|},
$$

где инфимум берется по всем конечным непустым подмножествам $A \subset G$ (см. [1]).

Известно, что группа аменабельна тогда и только тогда, когда $\mathrm{F}_{\varnothing}(G)=0$ для некоторого (а следовательно, для каждого) конечного порождающего множества $S$ (см. [2]-[4]). Напоминаем, что группа $G$ называется аменабельной, если для нее существует конечно-аддитивная мера $\mu$, определенная на $\sigma$-алгебре всех подмножеств группы $G$ и такая, что $\mu(G)=1$ и $\mu(g A)=\mu(A)$ для всяких $g \in G, A \subset G$. Как показано фон Нейманом [5], класс аменабельных групп замкнут относительно операций взятия подгруппы, факторгруппы, индуктивного предела, расширения. С другой стороны, любая группа, содержащая свободную подгруппу ранга 2, неаменабельна.

(C) В. С. Атавекян, 2009 
В 1977 г. Адяном была высказана гипотеза, что $m$-порожденные свободные периодические группы $B(m, n)$ нечетного периода $n \geqslant 665$ при $m \geqslant 2$ являются неаменабельными (см. [6]). Эта гипотеза была подтверждена им в работе [7]. Адяном в [7] найдено достаточное условие для неаменабельности групп, проблема равенства слов в которых решается алгоритмом Дэна (такие конечно-определенные группы называются гиперболическими (см. [8; теорема 1])). Далее, для относительно свободных групп $B(m, n)$ бернсайдова многообразия при $m \geqslant 2$ и нечетных $n \geqslant 665$ указана такая система определяющих соотношений, которая удовлетворяет как условию Дэна, так и указанному достаточному условию неаменабельности. Тем самым, в работе [7] впервые найдены неаменабельные группы, удовлетворяющие нетривиальному тождеству.

ОПРЕДЕЛЕНИЕ 2 (см. [1], [9]). Число

$$
\mathrm{F} \varnothing \mathrm{l}(G) \rightleftharpoons \inf _{S} \mathrm{~F} \mathrm{l}_{S}(G),
$$

где инфимум берется по всевозможным конечным порождающим множествам $S$ группы $G$, называется константой Фелнера группы $G$. Конечно-порожденная группа называется равномерно неаменабельной, если $\mathrm{F} \varnothing \mathrm{l}(G)>0$.

Известны некоторые классы равномерно неаменабельных групп. Например, любая неэлементарная гиперболическая группа, а также, любая широкая группа равномерно неаменабельна [1]. С другой стороны, существуют неаменабельные группы, которые не являются равномерно неаменабельными (см. [1], [10]).

В работе Осина [11] доказывается равномерная неаменабельность групп $B(m, n)$ при $m \geqslant 2$ и нечетных $n>10^{78}$. Этот результат ранее был получен в работе [1], но там доказательство опиралось на одну неподтвержденную пока гипотезу из работы [12].

Нашей основной целью является

ТЕОРема 1. Для каждого нечетного числа $n \geqslant 1003$ любая конечно-порожденная нециклическая подгруппа $H$ свободной бернсайдовой группь $B(m, n)$ - равномерно неаменабельная группа.

В частности, имеет место

СлеДСТВИЕ 1. Для любого $m \geqslant 2$ и нечетного $n \geqslant 1003$ свободная бернсайдовая группа $B(m, n)$ - равномерно неаменабельная группа.

Отметим, что как следует из хорошо известного результата П. Ноймана и Д. Уайголда, многообразия периодических групп показателя $n$ при $n \geqslant 3$ не являются шрейеровыми, т.е. не каждая подгруппа группы $B(m, n)$ является свободной $n$-периодической группой.

Как и в работах [1], [11], в доказательстве теоремы 1 существенно используется вышеуказанная теорема Адяна о неаменабельности групп $B(m, n)$.

Пусть $S$ - конечное порождающее множество группы $G$. Через $|g|_{S}$ обозначим минимальное целое число $n$ такое, что элемент $g \in G$ представляется в виде произведения $n$ элементов из множества $S \cup S^{-1}$. Для любого $n \geqslant 0$ множество

$$
B_{S}(n) \rightleftharpoons\left\{g \in G:|g|_{S} \leqslant n\right\}
$$


назовем $S$-шаром радиуса $n$. Количество элементов множества $B_{S}(n)$ обозначается через $\gamma_{S}(n)$. Поскольку $\gamma_{S}(m+n) \leqslant \gamma_{S}(m) \cdot \gamma_{S}(n)$, то $\gamma_{S}(n) \leqslant \gamma_{S}(1)^{n}$. Число

$$
\lambda(G, S)=\lim _{n \rightarrow \infty} \gamma_{S}(n)^{1 / n}
$$

называется степенъю экспоненциального роста группы $G$ относительно $S$.

ОПРЕДЕЛЕНИЕ 3. Если $\inf _{S} \lambda(G, S)>1$, где инфимум берется по всем конечным порождающим множествам $S$, то скажем, что группа $G$ имеет равномерный экспоненииальный рост.

Любая равномерно неаменабельная конечно порожденная группа имеет равномерный экспоненциальный рост (см. [1], [13]). Поэтому из теоремы 1 вытекает

СлеДСТВИЕ 2. Для любого $m \geqslant 2$ и нечетного $n \geqslant 1003$ любая конечно-порожденная нециклическая подгруппа $H$ свободной бернсайдовой группы $B(m, n)$ имеет равномерный экспоненииальный рост.

При $H=B(m, n)$ получаем

СлЕДСТВИЕ 3. Для любого $m \geqslant 2$ и нечетного $n \geqslant 1003$ свободная бернсайдовая группа $B(m, n)$ имеет равномерный экспоненциальный рост.

Следствием 3 для всех нечетных $n \geqslant 1003$ дается положительный ответ на вопрос де ля Арпа [14]: имеют ли бесконечные свободные бернсайдовые группы $B(m, n)$ равномерный экспоненциальный рост?

Из теоремы 4 работы Адяна [7] немедленно следует, что для любого натурального $\alpha \geqslant 1, m \geqslant 2$ и нечетного $n \geqslant 665$ вместе с группой $B(m, n)$ неаменабельна и группа $B(m, n, \alpha)$ (определение групп $B(m, n, \alpha)$ см. в [15; определение VI.2.2]). Поскольку $\mathrm{F} \varnothing \mathrm{l}(G) \geqslant \mathrm{F} \varnothing \mathrm{l}(G / N)$ для произвольной нормальной подгруппы $N$ группы $G$ (см. [1; теорема 4.1]), справедливо

СлеДСтвиЕ 4. Для любого натурального $\alpha \geqslant 1, m \geqslant 2$ и нечетного $n \geqslant 1003$ любая конечно порожденная нециклическая подгруппа $H$ группы $B(m, n, \alpha)$ - pавномерно неаменабельная группа.

При доказательстве теоремы 1 нам понадобится нижеследующая теорема, утверждающая существование такого числа $L$, что в каждом $S$-шаре радиуса $L$ содержатся два элемента, которые являются базисом свободной периодической подгруппы ранга 2 группы $B(m, n)$, где $S$ - произвольное множество, порождающее нециклическую подгруппу группы $B(m, n)$.

Теорема 2. Для любого $m \geqslant 2$ и нечетного $n \geqslant 1003$ существует такое число $L<(400 n)^{3}$, что для произвольного множества $S$, порождающего нециклическую подгруппу $\langle S\rangle_{B(m, n)}$ группы $B(m, n)$, можно найти элементы $u, v \in\langle S\rangle_{B(m, n)} m a-$ кие, что $\{u, v\}$ есть базис свободной бернсайдовой подгруппы периода $n$ и длинъ элементов $u, v$ относительно порождающего множества $S$ удовлетворяют неравенствам $|u|_{S}<L u|v|_{S}<L$.

Теорема 2 является усилением теоремы 2 [16] и теоремы 4 [17] автора. Похожее утверждение для неэлементарных гиперболических групп доказано в работе [18] Куби, где также показано, что неэлементарные гиперболические группы имеют равномерный экспоненциальный рост. Отметим, что при $m \geqslant 2$ и нечетных $n \geqslant 665$ 
группы $B(m, n)$ не могут быть заданы с помощью конечного числа определяющих соотношений (см. [15; теорема VI.2.13]), следовательно, не являются гиперболическими, хотя могут быть представлены как предел последовательности гиперболических групп (см. [19; теорема 7]).

1. Доказательство теоремы 2. Из теоремы Адяна VI.3.7 [15] непосредственно следует, что для любого нечетного $n \geqslant 665$ и конечного $m$ группа $B(m, n)$ изоморфно вкладывается в группу $B(2, n)$ (см. также [20]). Поэтому теорему 2 достаточно доказать в случае $m=2$. Пусть $S$ - произвольное множество, порождающее нециклическую подгруппу $\langle S\rangle_{B(2, n)}$ группы $B(2, n)$. Согласно теореме Адяна VI.3.3 [15] каждая абелевая подгруппа группы $B(m, n)$ циклическая, следовательно, подгруппа $\langle S\rangle_{B(2, n)}$ неабелевая. Поэтому можно выбрать пару неперестановочных элементов $X$ и $Y$ из множества $S$.

Лемма 1. Пусть $n \geqslant 1003$ - произвольное нечетное число. Тогда всякая неииклическая подгруппа $\Delta \rightleftharpoons\langle X, Y\rangle$ группь $B(2, n)$ содержит такую нециклическую подгруппу вида $U\langle A, C\rangle U^{-1}$, что $C$ - элементарный период некоторого ранга $\alpha$ u

$$
C \stackrel{B(m, n, \alpha-1)}{=}\left[A^{d}, Z^{-1} B^{d} Z\right]
$$

где $A$ и $B$ - минимизированные элементарные периоды некоторых рангов $\gamma u \beta$, $Z \in M_{\alpha-1}, \gamma \leqslant \beta \leqslant \alpha-1, d=191$ и длины слов $U A U^{-1}$ и $U C U^{-1}$ относительно порождающих $X$ и $Y$ удовлетворяют неравенствам

$$
\left|U A U^{-1}\right|_{\{X, Y\}}<(450 n)^{2} \quad u \quad\left|U C U^{-1}\right|_{\{X, Y\}}<(450 n)^{2} .
$$

ДокАЗАТЕЛЬСтво. Практически мы будем повторять доказательство леммы 7.3 из [21], попутно оценив длины слов для обоснования заключительной части леммы. Сначала заметим, что леммы $2.8,3.2,6.6$ и 7.2 из работы [21] остаются справедливыми, если в их формулировках и доказательствах эквивалентность в ранге $\alpha$ заменить на эквивалентность в ранге $\alpha$ в смысле монографии [15], а равенство слов в группе $\Gamma_{\alpha}$ заменить на равенство в группе $B(2, n, \alpha)$.

Пусть $\Delta \rightleftharpoons\langle X, Y\rangle_{B(2, n)}$ - произвольная нециклическая подгруппа группы $B(2, n)$. В силу VI.2.4 [15] и VI.1.2 [15]

$$
X \stackrel{B(2, n)}{=} T A^{i} T^{-1} \quad \text { и } \quad T^{-1} Y T \stackrel{B(2, n)}{=} Z^{-1} B^{j} Z
$$

для некоторых слов $T, Z$ и минимизированных элементарных периодов $A$ и $B$, имеющих ранги $\gamma$ и $\beta$. Без ограничения общности можем предположить, что $\gamma \leqslant \beta$, а в силу VI.2.4 [15] и IV.1.13 [15] можем считать, что $Z \in M_{\alpha} \cap A_{\alpha+1}$ для некоторого $\alpha \geqslant \beta$. Пусть НОД $(i, n)=k$ и $r$ - такое целое число, что $|r|<n$ и $A^{i r}=A^{k}$. Выбрав число $s \rightleftharpoons[n / 3 k]$, получим $n / 5<s k<n / 3$. Таким образом,

$$
X^{r s}=T A^{i r s} T^{-1}=T A^{k s} T^{-1}, \quad 186<k s<\frac{n+1}{2}-148,
$$

поскольку $n \geqslant 1003$. Итак, для слова $X_{1} \rightleftharpoons X^{r s}$ имеем

$$
X_{1}=T A^{k s} T^{-1}, \quad\left|X_{1}\right|_{\{X, Y\}} \leqslant|r s||X|_{\{X, Y\}}<\frac{n^{2}}{3} .
$$


Аналогично можно найти такой $Y_{1} \in\langle Y\rangle_{B(2, n)}$ и такие числа $t$ и $l$, что

$$
186<t l<\frac{n+1}{2}-148, \quad T^{-1} Y_{1} T=Z^{-1} B^{t l} Z, \quad\left|Y_{1}\right|_{\{X, Y\}}<\frac{n^{2}}{3} .
$$

По теореме VI.3.1 [15] $\left[X_{1}, Y_{1}\right] \neq 1$. В силу лемм 3.2 [21], 7.2 [21] и 2.8 [21] коммутатор

$$
\left[X_{1}, Y_{1}\right]=T\left[A^{k s}, Z^{-1} B^{t l} Z\right] T^{-1}
$$

сопряжен в $B(m, n)$ некоторому минимизированному элементарному периоду $D$ некоторого ранга $\delta \geqslant \beta+1$. Пусть

$$
T^{-1}\left[X_{1}, Y_{1}\right] T=Z_{1}^{-1} D Z_{1},
$$

где $Z_{1} \in M_{\lambda} \cap A_{\lambda+1}$ для некоторого $\lambda \geqslant \alpha$. Тогда, вновь применив леммы 3.2 [21], 7.2 [21] и 2.8 [21], получим, что коммутатор

$$
\left[X_{1},\left[X_{1}, Y_{1}\right]^{d}\right]=T\left[A^{k s}, Z_{1}^{-1} D^{d} Z_{1}\right] T^{-1}
$$

сопряжен в $B(2, n)$ некоторому минимизированному элементарному периоду $E$ некоторого ранга $\mu \geqslant \delta+1$. Допустим

$$
T^{-1}\left[X_{1},\left[X_{1}, Y_{1}\right]^{d}\right] T=Z_{2}^{-1} E Z_{2} .
$$

Таким образом, в подгруппе $\Delta \rightleftharpoons\langle X, Y\rangle_{B(2, n)}$ содержатся элементы

$$
\left[X_{1}, Y_{1}\right]=T Z_{1}^{-1} D Z_{1} T^{-1} \quad \text { и } \quad\left[X_{1},\left[X_{1}, Y_{1}\right]^{d}\right]=T Z_{2}^{-1} E Z_{2} T^{-1} .
$$

Можно считать, что $Z_{3}^{-1} \rightleftharpoons Z_{1} Z_{2}^{-1} \in M_{\nu} \cap A_{\nu+1}$, где $\nu \geqslant \mu$. По лемме 3.2 [21] найдем приведенную форму $C$ коммутатора $\left[D^{d}, Z_{3}^{-1} E^{d} Z_{3}\right]$. Согласно лемме $7.2[21] C$ элементарный период некоторого ранга $\tau \geqslant \mu+1$. В силу 3.6 [21]

$$
C \stackrel{B(2, n, \mu)}{=} w\left[D^{d}, Z_{3}^{-1} E^{d} Z_{3}\right] w^{-1}, \quad \text { где } \quad w \in \Theta\left(D, D_{1}\right) .
$$

Рассмотрим элементарные периоды $A \rightleftharpoons w D w^{-1}$ и $C=w\left[D^{d}, Z_{3}^{-1} E^{d} Z_{3}\right] w^{-1}$. Из определений следует, что

$$
\begin{aligned}
& A=w Z_{1} T^{-1}\left[X_{1}, Y_{1}\right] T Z_{1}^{-1} w^{-1}, \\
& C=w Z_{1} T^{-1}\left[\left[X_{1}, Y_{1}\right]^{d},\left[X_{1},\left[X_{1}, Y_{1}\right]^{d}\right]^{d}\right] T Z_{1}^{-1} w .
\end{aligned}
$$

Значит, если $U \rightleftharpoons T Z_{1}^{-1} w^{-1}$, то $U A U^{-1} \in \Delta, U C U^{-1} \in \Delta$ и

$$
\begin{aligned}
\left|U A U^{-1}\right|_{\{X, Y\}} & =\left|\left[X_{1}, Y_{1}\right]\right|_{\{X, Y\}}<\frac{n^{2}}{3}|[X, Y]|_{\{X, Y\}}=\frac{4}{3} n^{2} \\
\left|U C U^{-1}\right|_{\{X, Y\}} & =\left|\left[\left[X_{1}, Y_{1}\right]^{d},\left[X_{1},\left[X_{1}, Y_{1}\right]^{d}\right]^{d}\right]\right|_{\{X, Y\}}<\frac{n^{2}}{3}(8 d+2 d(8 d+2)) .
\end{aligned}
$$

Остается заметить, что

$$
\frac{n^{2}}{3}(8 d+2 d(8 d+2))<(450 n)^{2}
$$

Лемма доказана. 
Следующее утверждение доказано автором в работе [16].

Лемма 2 (теорема $2[16])$. Пусть коммутатор $\left[A^{d}, Z^{-1} B^{d} Z\right]$ в групnе $B(2, n$, $\alpha-1)$ равен элементарному периоду $C$ ранга $\alpha$, где $A$ - элементарный период ранга $\gamma, B$ - элементарный период ранга $\beta, Z \in M_{\alpha-1}, \gamma \leqslant \beta \leqslant \alpha-1, d=191, n \geqslant$ 1003 - произвольное нечетное число и слова $A^{q}$ и $B^{q}$ входят в некоторые слова из множеств $M_{\gamma-1}$ и $M_{\beta-1}$ соответственно. Тогда слова

$$
u \rightleftharpoons C^{200} A C^{200} A^{2} \cdots A^{n-1} C^{200}, \quad v \rightleftharpoons C^{300} A C^{300} A^{2} \cdots A^{n-1} C^{300}
$$

являются базисом свободной бернсайдовой подгруппы ранга 2 группы $B(2, n)$.

Перейдем к доказательству теоремы 2.

ДОКАЗАТЕЛЬСТВО ТЕОРЕМЫ 2. Поскольку подгруппы

$$
\langle u, v\rangle_{B(2, n)} \quad \text { и } \quad\left\langle U u U^{-1}, U v U^{-1}\right\rangle_{B(2, n)}
$$

изоморфны, в силу леммы 2 элементы $U u U^{-1}, U v U^{-1}$ являются базисом свободной бернсайдовой подгруппы ранга 2 группы $B(2, n)$. Очевидно,

$$
\begin{aligned}
& U u U^{-1}=\left(U C U^{-1}\right)^{200}\left(U A U^{-1}\right) \cdots\left(U A U^{-1}\right)^{n-1}\left(U C U^{-1}\right)^{200}, \\
& U v U^{-1}=\left(U C U^{-1}\right)^{300}\left(U A U^{-1}\right) \cdots\left(U A U^{-1}\right)^{n-1}\left(U C U^{-1}\right)^{300} .
\end{aligned}
$$

Согласно лемме 1 имеем $U u U^{-1}, U v U^{-1} \in\langle X, Y\rangle_{B(m, n)}$. Используя неравенства (1) и (2), получаем

$$
\begin{aligned}
& \left|U u U^{-1}\right|_{\{X, Y\}}<\frac{n(n-1)}{2} 4 n^{2}+200 n(450 n)^{2}, \\
& \left|U v U^{-1}\right|_{\{X, Y\}}<\frac{n(n-1)}{2} 4 n^{2}+300 n(450 n)^{2} .
\end{aligned}
$$

Заметим, что

$$
\left|U u U^{-1}\right|_{S} \leqslant\left|U u U^{-1}\right|_{\{X, Y\}}, \quad\left|U v U^{-1}\right|_{S} \leqslant\left|U v U^{-1}\right|_{\{X, Y\}},
$$

поскольку $\{X, Y\} \subseteq S$. В качестве $L$ можно взять число $2 n^{2}(n-1)+300 n(450 n)^{2}<$ $(400 n)^{3}$. Теорема 2 доказана.

2. Доказательство теоремы 1. Следующая лемма доказана в работе [1].

ЛЕмма 3 (теорема 7.1 [1]). Пусть $G$ - конечно-порожденная группа с множеством порождающих $S=\left\{x_{1}, \ldots, x_{s}\right\}$, a $H$ - подгруппа группы $G$ с множеством порождающих $S^{\prime}=\left\{y_{1}, \ldots, y_{k}\right\}$. Обозначим через $L$ максимальную длину элементов $y_{1}, \ldots, y_{k}$ относительно порождающих $S=\left\{x_{1}, \ldots, x_{s}\right\}$. Тогда

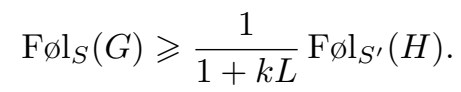

ЛЕмма 4. Пусть $G$ - конечно-порожденная группа с множеством порождаю-

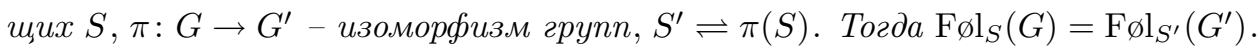

ДокАзАТЕЛЬство очевидно (см. также теорему $4.1[1])$. 
Чтобы доказать теорему 1 , предположим, что $S$ есть произвольное конечное множество порождающих элементов нециклической подгруппы $H$ группы $B(m, n)$. По теореме 2 существуют элементы $u, v \in H$ такие, что $\{u, v\}$ есть базис свободной бернсайдовой подгруппы ранга 2 и длины элементов $u, v$ относительно порождающего множества $S$ удовлетворяют неравенствам $|u|_{S}<L$ и $|v|_{S}<L$, где число $L$ не зависит от выбора множества $S$. Согласно лемме 3 имеем

$$
\mathrm{F}_{\varnothing}(H) \geqslant \frac{1}{1+2 L} \mathrm{~F}_{S} \mathrm{l}_{\{u, v\}}\left(\langle u, v\rangle_{B(m, n)}\right) .
$$

В силу леммы 4 число $b \rightleftharpoons \mathrm{F}_{\{u, v\}}\left(\langle u, v\rangle_{B(m, n)}\right)$ не зависит от выбора пары свободных порождающих $u, v$. Поскольку по теореме Адяна (теорема 5 работы [7]) группа $B(2, n)$ - неаменабельная, $b>0$. Таким образом, для любого множества порождающих $S$ нециклической подгруппы $H$ имеем

$$
\mathrm{F}_{\varnothing} \mathrm{l}_{S}(H) \geqslant \frac{b}{1+2 L}>0
$$

из чего следуют неравенства

$$
\mathrm{F} \varnothing \mathrm{l}(H) \rightleftharpoons \inf _{S} \mathrm{~F}_{\varnothing l}(H) \geqslant \frac{b}{1+2 L}>0 .
$$

Теорема 1 доказана.

\section{СПИСОК ЦИТИРОВАННОЙ ЛИТЕРАТУРЫ}

[1] G. N. Arzhantseva, J. Burillo, M. Lustig, L. Reeves, H. Short, E. Ventura, "Uniform non-amenability", Adv. Math., 197:2 (2005), 499-522.

[2] E. Følner, "On groups with full Banach mean value", Math. Scand., 3 (1955), 243-254.

[3] I. Namioka, "Følner's condition for amenable semi-groups", Math. Scand, 15 (1964), 18-28.

[4] A. Hulanicki, "Means and Følner condition on locally compact groups", Studia Math., 27 (1966), 87-104.

[5] J. von Neumann, "Zur algemeinen theorie des masses", Fund. Math., 13 (1929), 73-116.

[6] С.И. Адян, "Аксиоматический метод построения групп с заданными свойствами", УМН, 32:1 (1977), 3-15.

[7] С. И. Адян, "Случайные блуждания на свободных периодических группах", Изв. АН СССР. Сер. матем., 46:6 (1982), 1139-1149.

[8] И.Г. Лысёнок, "О некоторых алгоритмических свойствах гиперболических групп", Изв. АН СССР. Сер. матем., 53:4 (1989), 814-832.

[9] P. de la Harpe, A. Valette, "La propriété (T) de Kazhdan pour les groupes localement compacts (avec un appendice de Marc Burger)", Astérisque, 175, Soc. Math. France, Paris, 1989.

[10] D. V. Osin, "Weakly amenable groups", Computational and Statistical Group Theory (Las Vegas, NV/Hoboken, NJ, 2001), Contemp. Math., 298, Amer. Math. Soc., Providence, RI, 2002, 105-113.

[11] D. V. Osin, "Uniform non-amenability of free Burnside groups", Arch. Math. (Basel), 88:5 (2007), 403-412.

[12] S. V. Ivanov, A. Yu. Ol'shanskii, "Some applications of graded diagrams in combinatorial group theory", Groups, V. 2 (Proc. Int. Conf., St. Andrews, UK 1989), London Math. Soc. Lecture Note Ser., 160, Cambridge Univ. Press, Cambridge, 1991, 258-308.

[13] Y. Shalom, "Explicit Kazhdan constants for representations of semisimple and arithmetic groups", Ann. Inst. Fourier (Grenoble), 50:3 (2000), 833-863. 
[14] P. de la Harpe, "Uniform growth in groups of exponential growth", Geom. Dedicata, 95:1 (2002), 1-17.

[15] С. И. Адян, Проблема Бернсайда и тождества в группах, Наука, М., 1975.

[16] В. С. Атабекян, "О подгруппах свободных периодических групп нечетного периода $n \geqslant 1003$ ”, Изв. РАН. Сер. матем. (в печати).

[17] В.С. Атабекян, "О простых и свободных периодических группах", Вестн. Моск. ун-та. Сер. 1. Матем., мех., 1987, №6, 76-78.

[18] M. Koubi, "Croissance uniforme dans les groupes hyperboliques", Ann. Inst. Fourier (Grenoble), 48:5 (1998), 1441-1453.

[19] С.И. Адян, "Проблема Бернсайда о периодическоих группах и смежные вопросы", Современные проблемы математики, Вып. 1, МИАН, М., 2003, 5-29.

[20] В. Л. Ширванян, "Вложение группы $\mathbf{B}(\infty, n)$ в группу $\mathbf{B}(2, n)$ ", Изв. АН СССР. Сер. матем., 40:1 (1976), 190-208.

[21] С. И. Адян, И. Г. Лысёнок, "О группах, все собственные подгруппы которых конечные циклические", Изв. АН СССР. Сер. матем., 55:5 (1991), 933-990.

В. С. Атабекян

Ереванский государственный университет

E-mail: avarujan@ysu.am
Поступило

22.04 .2008

Исправленный вариант

30.06 .2008 